\title{
The cause of the raised plasma urea of acute heart failure
}

\author{
R. D. THOMAS \\ M.R.C.P.
}

\author{
Alison Newill
}

A.I.M.L.S.

\author{
D. B. Morgan \\ M.R.C.Path.
}

Departments of Cardiology and Chemical Pathology, Leeds General Infirmary

\begin{abstract}
Summary
The concentrations of urea, urate, phosphate and creatinine were measured in the plasma of 30 consecutive patients admitted acutely with heart failure. On admission, 20 had a raised plasma urea, 21 had a raised plasma urate, but only 6 had a raised plasma phosphate and only 6 had a raised plasma creatinine. A further 9 of the patients developed a raised plasma urea after admission. The increase in plasma urea present on admission was greater than expected for the fall in GFR (as indicated by the increase in plasma creatinine). The results for plasma and urine taken together suggest that a major cause of the raised plasma urea was an increased urea production rather than a reduced glomerular filtration rate. There was no obvious relationship between plasma urea and clinical features, or diuretic therapy.
\end{abstract}

\section{Introduction}

A raised plasma urea is common in heart failure (Domenet and Evans, 1969; Thayer, 1960) and the higher it is, the lower the chances of survival (Domenet and Evans, 1969; Morgan, Carver and Payne, 1977). This rise is usually attributed to diminished renal function (Thayer, 1960), or to treatment with diuretics (Greenblatt et al., 1977). However, in a recent survey, most hospital patients with a raised plasma urea and heart failure, had a normal plasma creatinine and, therefore, presumably had normal renal function (Morgan et al., 1977). Apart from diminished renal function the other two possible causes of a raised plasma urea are an increased production of urea and increased tubular reabsorption of urea. Increased urea production has been demonstrated in heart failure (Domenet and Evans, 1969; Ujjwal, Bhatnagar and Bordia, 1974) but its relative contribution to the raised plasma urea has not been assessed. The authors have attempted to define the contribution of these three

Correspondence to: Professor D. B. Morgan, Department of Chemical Pathology, The Martin Wing, The General Infirmary, Leeds LS1 3EX. causes to the raised plasma urea in patients admitted acutely to hospital with heart failure. In addition they have examined the relationships between plasma urea and plasma urate, phosphate and creatinine in these patients on admission, and made observations on the changes in plasma urea while the patients were in hospital.

\section{Patients and methods}

Thirty-two consecutive patients who were admitted acutely to hospital because of heart failure were studied. The diagnosis was based on a typical history and clinical signs together with radiological evidence of pulmonary venous hypertension. Two patients were subsequently shown to have chronic renal failure and were excluded. There were 19 men and 11 women aged 42-92 years (mean 69 years). The major cause of the heart failure was ischaemic heart disease in 13 patients, cor pulmonale in 6 , valvular disease in 5, cardiomyopathy in 1 and chronic heart block in 1 patient. Four elderly patients had no obvious single cause for the heart failure. Twenty-eight of the $\mathbf{3 0}$ patients were being treated for heart failure before admission. The clinical signs and the radiological features were graded on a simple scale of severity. A sample of blood and an untimed sample of urine were taken within a day of admission. Further measurements were made during the admission on blood samples taken for clinical management. The results were compared with those in 36 patients with chronic heart disease (group CHD) who were attending outpatients or who had been admitted for catheterization (Davidson et al., 1976).

Plasma urea, phosphate, and creatinine were measured with a Vickers M300 Multichannel Analyser. Plasma urate was measured by the standard AutoAnalyser technique (N13A). Urine urea and creatinine were measured with a Vickers D300 Dualchannel Analyser. The ratio of urea to creatinine in the urine $(\mathrm{U} / \mathrm{Cr})$ was taken as a measure of urea excretion rate and thus of urea production rate. The percentage tubular resorption of urea 

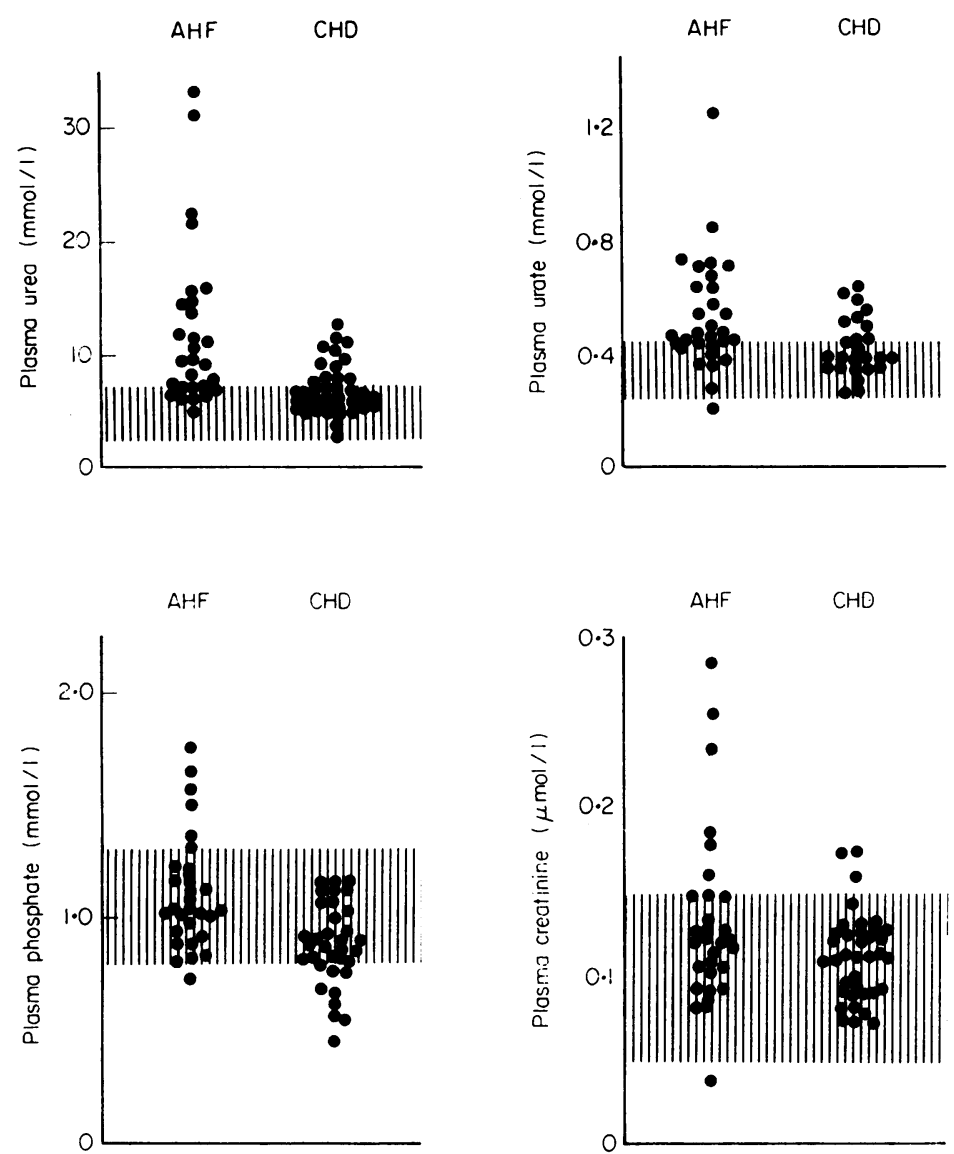

Fig. 1. The plasma urea, urate phosphate and creatinine in the patients admitted acutely with heart failure (AHF) and the patients with chronic heart disease (CHD). The hatched areas indicate the reference ranges of the Leeds General Hospital laboratory.

$(\% R)$ was calculated from the concentrations of urea and creatinine in urine and plasma;

$$
\% R=\left[1-\frac{U_{u}}{U_{p}} \cdot \frac{C_{p}}{C r_{u}}\right] \times 100
$$

where $U_{p}, U_{u}$ and $C r_{p}$ and $\mathrm{Cr}_{u}$ are the concentrations $(\mathrm{mmol} / \mathrm{l})$ of urea and creatinine in plasma and urine respectively.

\section{Results}

Figure 1 shows the distributions of plasma urea, urate, phosphate and creatinine in the 30 patients with heart failure (AHF) and in the 36 patients with chronic heart disease (CHD). The plasma urea was above the upper limit of normal $(7 \cdot 1 \mathrm{mmol} / \mathrm{l})$ in $20(67 \%)$ of the patients in group AHF and in 14 $(39 \%)$ of the patients in group CHD and, whereas
$6(20 \%)$ of group AHF had a plasma urea greater than $15 \mathrm{mmol} / 1$, there were no such values in group CHD. The plasma urate was above the upper limit of normal $(0.45 \mathrm{mmol} / \mathrm{l})$ in $20(67 \%)$ of the patients with AHF and $14(38 \%)$ of the patients with CHD and the frequency of raised values of plasma phosphate $(>1.3 \mathrm{mmol} / \mathrm{l})$ was $20 \%$ in group AHF but zero in group CHD. The plasma creatinine was above the upper limit of normal $(150 \mu \mathrm{mol} / \mathrm{l})$ in only 6 $(20 \%)$ of the patients in group AHF and only 3 $(8 \%)$ of patients in group CHD. Fourteen of the patients with a raised plasma urea had a plasma creatinine within the normal range.

The initial plasma urea in patients in group AHF was not related to the age of the patient, the cause of the heart failure or the degree of cardiomegaly or pulmonary venous hypertension of the chest X-ray. Although 2 patients with a systolic blood pressure less than $100 \mathrm{mmHg}$ had the highest values of 

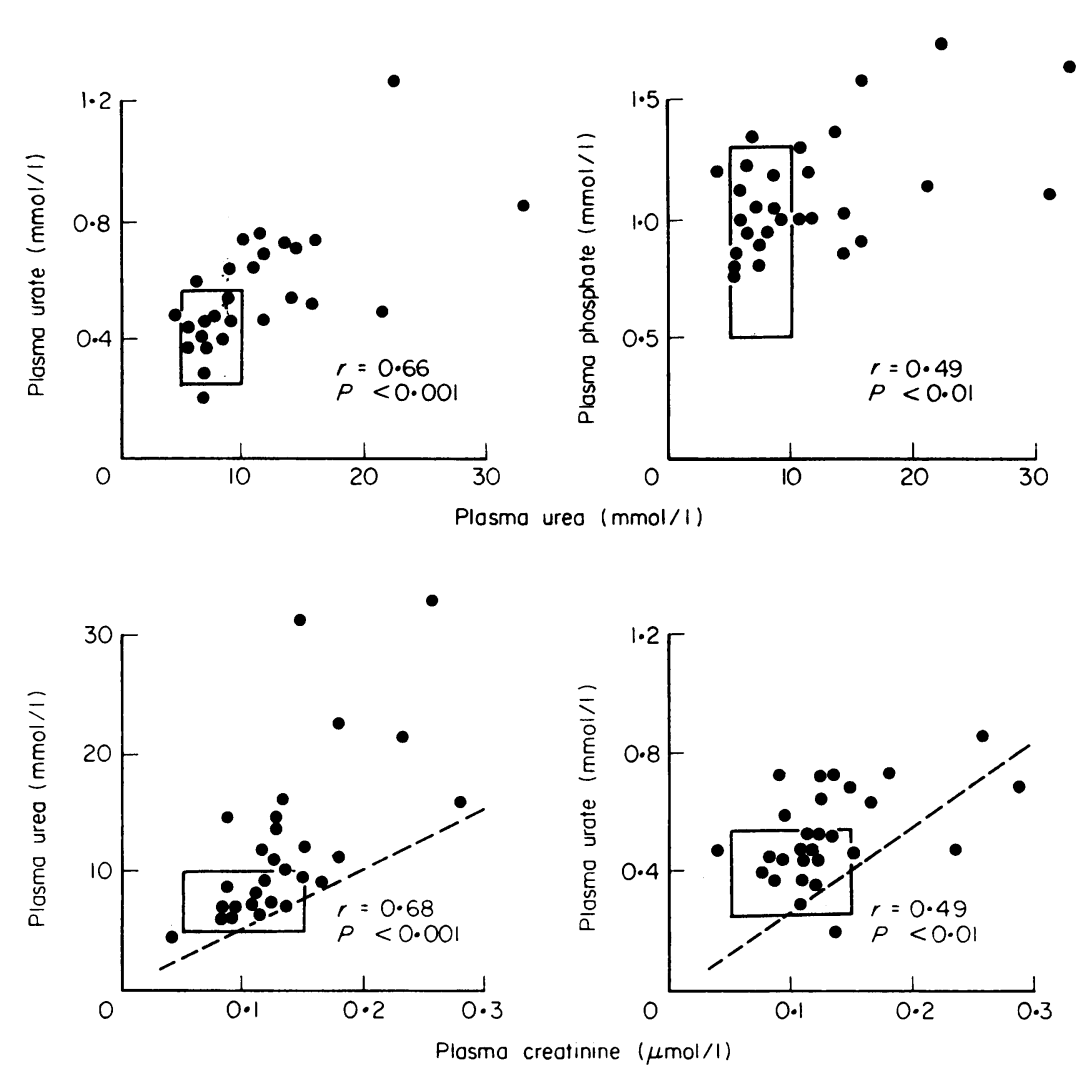

Fig. 2. The relation between plasma urate and urea, phosphate and urea, urea and creatinine and urate and creatinine in the patients with AHF. The values in the patients with CHD were all within the box and are not shown individually. The lines indicate the relation between the mean values which would be expected on the basis of a fall in GFR alone.

plasma urea, there was no other relationship with its severity of the clinical signs of heart failure.

Figure 2 shows that in group AHF there were significant correlations between plasma urea and each of plasma urate, phosphate and creatinine and between plasma urate and plasma creatinine. However, the increase in plasma urea and plasma urate was greater than would be expected from a fall in GFR alone (as indicated by the rise in plasma creatinine), which would be a doubling of the plasma urea (and urate) for a halving of GFR (doubling of the plasma creatinine).

The 2 determinants of plasma urea, other than GFR, are the rate of production of urea (which have been assessed by the urine $\mathrm{U} / \mathrm{Cr}$ ) and the tubular resorption of urea. Twenty-seven patients had urine collections and Fig. 3 shows that one-third of the patients with AHF had a urine $\mathrm{U} / \mathrm{Cr}$ above the highest value in group CHD. In contrast, the tubular resorption of urea was the same in the 2 groups. The rise in plasma urea in the patients with AHF compared with those with CHD was about equally due to an increase in urea excretion and a fall in GFR, the plasma urea was increased by a factor of 1.9 on the average, and the proportional increases due to increase in urea excretion and a fall in GFR were 1.5 and 1.4 respectively. There was no correlation between the contribution from the 2 causes.

Further measurements were made in 28 patients. Of the 11 patients whose plasma urea was normal on admission, 9 developed a rise above normal during the stay in hospital. Plasma creatinine also increased in these patients but it did not exceed the upper limit of normal. There was a general trend for the plasma urea to rise and then fall during admission. This subsequent fall was not related to a reduction in the dose of diuretic therapy. 

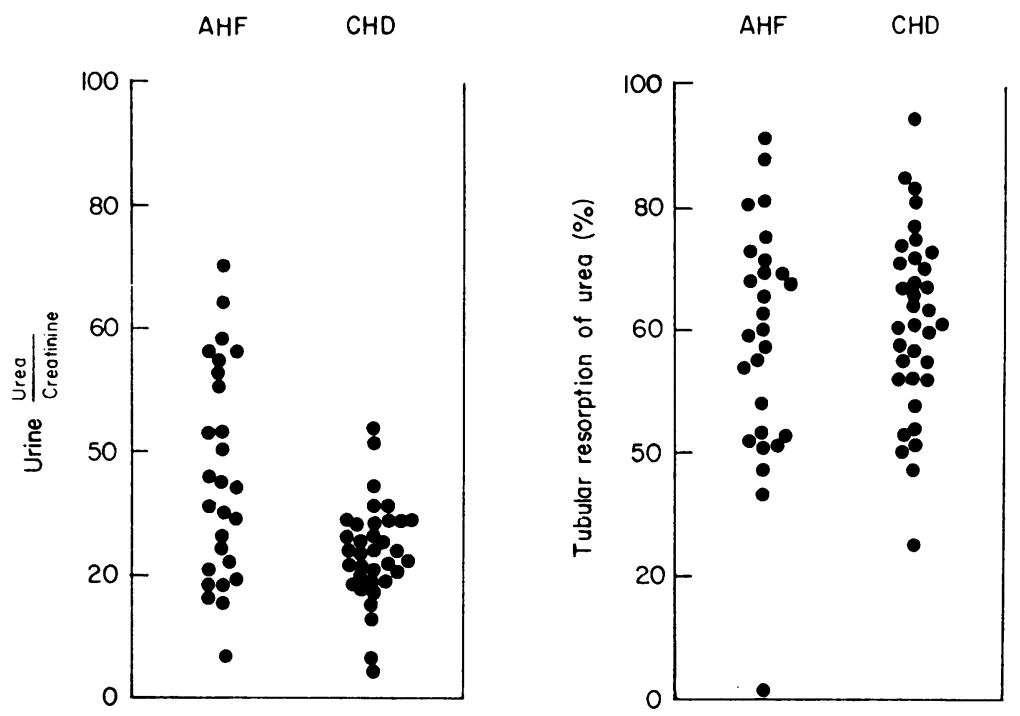

FIG. 3. The urine urea : creatinine ratio and the percentage tubular resorption of urea in the groups AHF and CHD.

\section{Discussion}

In patients with chronic heart disease the frequency of a raised plasma urea was $39 \%$, and this is similar to 2 other series (Thayer, 1960; Ujjwal et al., 1974). In the present series, $67 \%$ of the patients with acute heart failure had a raised plasma urea on admission to hospital and the frequency increased to $94 \%$ for the whole admission. The frequency of a raised urate was also high $(69 \%)$ while that for phosphate and creatine was lower $(20 \%)$.

The plasma urea, urate and phosphate were directly related to the plasma creatinine in these patients. Since raised values of these measurements are well recognized features of renal failure this might suggest that the rise in plasma urea is due to impaired renal function (Thayer, 1960; Fishberg, 1947). However, Domenet and Evans (1969), in a large clinical survey, suggested that the increase in plasma urea was largely due to an increased production of urea. A third possible explanation is that there is an increased tubular resorption of urea, and this has not been previously considered.

The present authors found that the plasma urea was higher than would be expected for an uncomplicated fall in GFR and, in 14 patients with a raised urea, the creatinine was normal. This extra rise was caused by an increased production of urea and not to an increased tubular resorption of urea. In the group as a whole, the contribution from a fall in GFR was equal to that from an increase in urea production, but these factors were not cor- related. It was not possible to define the exact contribution in any individual patient, from these factors because of the wide range of normal values.

The association between the administration of diuretics and the rise in plasma urea in heart failure is often assumed to be causal and due to volume depletion (Greenblatt et al., 1977). However, in most of the patients in the present series in whom the plasma urea returned towards normal in hospital the dose of diuretics was either maintained or even increased and in only 2 patients did it appear that volume depletion had a significant role. It seems likely that the contribution of diuretic therapy to the raised plasma urea of heart failure has been exaggerated. This study suggests that a raised plasma urea, which is a very common event in acute heart failure, should not be assumed to be due to impaired renal function or to treatment with diuretics. Although a fall in GFR undoubtedly contributes, an equally important factor for the increase in the plasma urea is increased urea production, probably from increased protein breakdown. This protein breakdown could be regarded as part of the metabolic response to acute stress or trauma rather than a consequence of circulatory failure, and in the long term could explain the muscle wasting of heart failure.

\section{Acknowledgments}

We wish to thank the many physicians who allowed us to study their patients, and the many staff who helped us. Dr C. Davidson kindly gave us permission to include unpublished data from a previous study. 


\section{References}

Davidson, C., Burkinshaw, L., Mclachlan, M.S.F. \& MorgaN, D.B. (1976) Effect of long-term diuretic treatment on body-potassium in heart disease. Lancet, ii, 1044.

DomeneT, J.G. \& Evans, D.W. (1969) Uraemia in congestive heart failure. Quarterly Journal of Medicine, 149, 117.

FishberG, A.M. (1947) Hypertension and Nephritis. Lea \& Febiger, Philadelphia.

Greenblatt, D.J., Duhme, D.W., Allen, M.D. \& KochWESER, J. (1977) Clinical toxicity of furosemide in hos- pitalized patients. A report from the Boston Collaborative Drug Surveillance Program. American Heart Journal, 94, 6. Morgan, D.B., Carver, M.E., \& Payne, R.B. (1977) Plasma creatinine and urea : creatinine ratio in patients with raised plasma urea. British Medical Journal, 2, 929.

THAYER, J.M. (1960) Renal abnormalities in congestive heart failure. Stanford Medical Bulletin, 18, 129.

UjJwal, J.S., Bhatnagar, H.N.S. \& Bordia, A. (1974) Uraemia in congestive heart failure. Journal of Association of Physicians of India, 22, 589. 